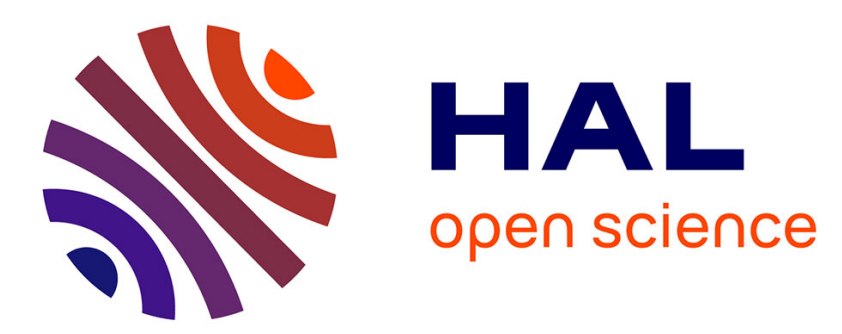

\title{
Reliability of task graph schedules with transient and fail-stop failures: complexity and algorithms
} Anne Benoit, Louis-Claude Canon, Emmanuel Jeannot, Yves Robert

\section{To cite this version:}

Anne Benoit, Louis-Claude Canon, Emmanuel Jeannot, Yves Robert. Reliability of task graph schedules with transient and fail-stop failures: complexity and algorithms. Journal of Scheduling, 2012, 15 (5), pp.615-627. 10.1007/s10951-011-0236-y · hal-00653477

\section{HAL Id: hal-00653477 https://hal.inria.fr/hal-00653477}

Submitted on 19 Dec 2011

HAL is a multi-disciplinary open access archive for the deposit and dissemination of scientific research documents, whether they are published or not. The documents may come from teaching and research institutions in France or abroad, or from public or private research centers.
L'archive ouverte pluridisciplinaire HAL, est destinée au dépôt et à la diffusion de documents scientifiques de niveau recherche, publiés ou non, émanant des établissements d'enseignement et de recherche français ou étrangers, des laboratoires publics ou privés. 


\title{
Reliability of task graph schedules with transient and fail-stop failures: complexity and algorithms
}

\author{
Anne Benoit · Louis-Claude Canon · Emmanuel Jeannot · Yves Robert
}

Received: date / Accepted: date

\begin{abstract}
This paper deals with the reliability of task graph schedules with transient and fail-stop failures. While computing the reliability of a given schedule is easy in the absence of task replication, the problem becomes much more difficult when task replication is used. We fill a complexity gap of the scheduling literature: our main result is that this reliability problem is \#P'-Complete (hence at least as hard as NP-Complete problems), both for transient and for failstop processor failures. We also study the evaluation of a restricted class of schedules, where a task cannot be scheduled before all replicas of all its predecessors have completed their execution. Although the complexity in this case with fail-stop failures remains open, we provide an algorithm to estimate the reliability while limiting evaluation costs, and we validate this approach through simulations.
\end{abstract}

Keywords Complexity results, algorithms, task graph schedules, reliability, fail-stop and transient failures.

\section{Introduction}

Since the landmark papers of Bannister and Trivedi (1983), Shatz et al (1992) and Kartik and Murthy (1997), numerous papers have dealt with reliability issues in task graph scheduling. A natural approach to cope with processor failures is to use redundancy for critical parts of the applica-

Anne Benoit · Yves Robert

LIP, ENS Lyon, 46 Allée d'Italie, 69364 Lyon Cedex 07, France, and Institut Universitaire de France, E-mail: Anne.Benoit@ens-lyon.fr, Yves.Robert@ens-lyon.fr

Louis-Claude Canon

Nancy University, 34 Cours Léopold, CS 25233, 54052 Nancy Cedex, France and INRIA, E-mail: Louis-Claude.Canon@inria.fr

Emmanuel Jeannot

LaBRI and INRIA Bordeaux, 351 Cours de la Libération - Bât. A29b, 33405 Talence Cedex, France, E-mail: Emmanuel.Jeannot@inria.fr tion (Barlow and Proschan 1967), which in the scheduling framework amounts to replicate the execution of some (or all) tasks. Replication will increase the probability that the execution is successful: only one successful copy of each task is needed when one or several failures take place during the execution. However, one must be able to evaluate the reliability of a given schedule with replication, in order to compare different possible mappings.

We note that at the application level, checkpoint/restart strategies are commonly used as another approach to recover from failures (Cappello et al 2009). Such mechanisms may turn out very costly, depending on the size of the application image, and the number of resources enrolled for execution. In any case, checkpointing is complementary to replication and these techniques do no exclude each other. In both cases, it is necessary to provide an optimized mapping of the application that minimizes the probability of failure.

In this paper, we focus on the problem of computing the reliability of a schedule, i.e., the probability that its execution is successful. More precisely, we are given a schedule that executes an application task graph on a parallel system, and that executes some tasks more than once to achieve redundancy. Moreover, each scheduled task has a known probability of failure. An example of such a schedule is given on Fig. 1.

This problem has been partially addressed in the literature. It is known that if replication is not allowed, then the problem has a polynomial-time algorithm (Dongarra et al 2007; Jeannot et al 2008). A recent paper recognizes that it is difficult to compute the reliability of a schedule with replication, and proposes exponential time algorithms (Girault and Kalla 2009). To the best of our knowledge, the complexity of the problem with replication has never been established. We fill this complexity gap and show that the problem is indeed \#P'-Complete, hence at least as hard as NP-Complete problems. The complexity class \#P' is a natural extension of 


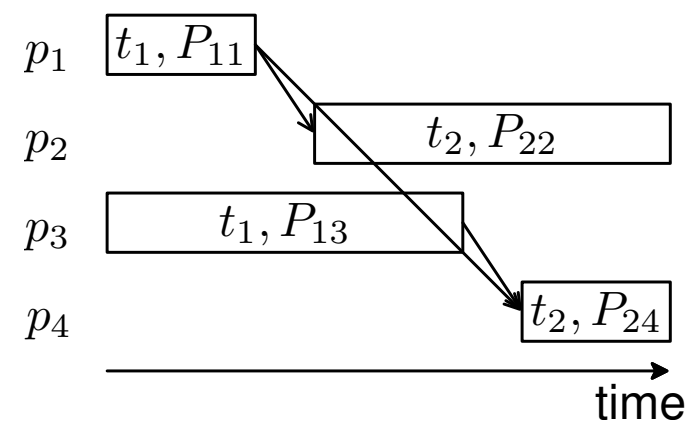

Fig. 1: General schedule of a chain of two tasks ( $t_{1}$ and $\left.t_{2}\right)$ that are duplicated twice each. $P_{i j}$ is the failure probability of task $t_{i}$ on processor $p_{j}$.

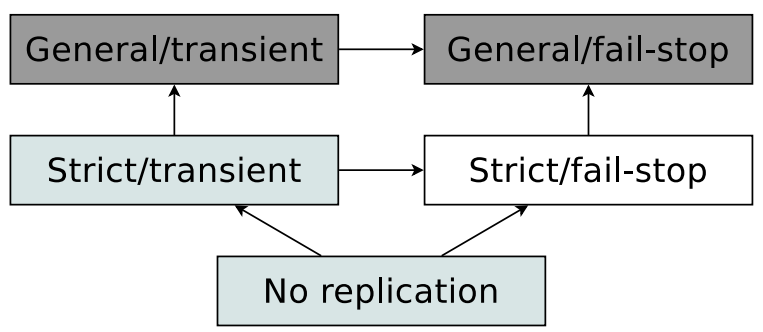

Fig. 2: Summary of complexity results of this paper. White: open problem. Light grey: solvable in polynomial time. Dark grey: \#P'-Complete. Arrow: polynomial-time reduction.

\#P, the class of counting problems corresponding to NP decision problems (Bodlaender and Wolle 2004), in which we can apply a polynomial function on the \#P integer output (we need a rational number for the reliability).

There are two major failure types, transient and failstop. In a nutshell, transient failures invalidate only the execution of the current task, and the processor subject to that failure will be able to recover and execute the subsequent tasks assigned to it (if any). On the contrary, fail-stop failures are unrecoverable: once the fault occurs, the corresponding processor is down until the end of the whole execution.

We further explore a particular class of schedules, which we call strict schedules. Strict schedules obey a simple rule, called replication for reliability in Girault et al (2009): if there is a dependence edge from task $t$ to task $t^{\prime}$ in the task graph, then all replicas of $t$ must complete their executions before any replica of $t^{\prime}$ can start its execution. As only one replica of $t$ needs to complete its execution before one of the replicas of $t^{\prime}$ starts its execution in a feasible schedule, it guarantees more precedence constraints than necessary. Schedules for which this rule is not enforced are called general. Fig. 1 is an example of such a general schedule.

With two failure types and two schedule classes, we are led to four variations of the problem. Fig. 2 summarizes known results on the complexity of reliability evaluation for these variations, with the following legend: light grey for polynomial time, white for open, and dark grey for \#P'Complete. An arrow means that the source problem is polynomial-time reducible to the destination problem. The major contribution of the paper is the \#P'-Completeness of the problem for general schedules, for both failure types.

Another contribution of the paper is to provide a new approach to estimate the reliability of strict schedules. In the case of transient failures, it is known in the literature that evaluating the reliability is a polynomial-time problem: scheduling task graphs without replication has been studied in Dongarra et al (2007) and Jeannot et al (2008), while the case with replication is studied in Girault et al (2009). However, we are not aware of polynomial-time techniques to compute the reliability of strict schedules in the presence of fail-stop failures. The proposed approach applies to any strict schedule and is empirically validated on random instances.

The paper is organized as follows. We briefly overview related work on \#P-Complete problems in Section 2. Then we present the models in Section 3, together with a little example intended to help understand the difficulty of computing the reliability of a schedule. The core contribution, namely the \#P'-Completeness of reliability evaluation, is presented in Section 4. Section 5 is devoted to results for strict schedules. Finally, we conclude in Section 6.

\section{Related work on \#P-Complete problems}

In some related work (Valiant 1979), Valiant proves that computing the number of acceptable solutions for the two terminal problem is \#P-Complete. The work of Provan and Ball (1983) extends the above result for the case of DAGs, and shows that evaluating the probability of success in the two terminal case is \#P'-Complete. However, their result does not imply anything about the complexity of the schedule reliability problem. Furthermore, it is interesting to remark that evaluating the reliability of a system is often performed through Reliability Block Diagrams (RBD) (Bream 1995). It is assumed in many papers such as in Girault et al (2009) that RBD evaluation has an exponential time. However, to the best of our knowledge, there is no formal complexity result to support this claim. Although it is possible to show that RBD evaluation is also \#P'-Complete from Provan and Ball (1983), we can easily derive it from our results. However, in some cases, RBDs may have a special structure that allows for an evaluation in polynomial time. 


\section{Framework}

Our main objective is to study the reliability of different types of schedules. First, we formalize the execution model by detailing the application and platform parameters in Section 3.1. Then, we characterize in Section 3.2 the failure model that specifies how processors may fail during the execution of any task. Next, we describe in Section 3.3 the replication mechanism consisting in scheduling some tasks several times. We are then able to provide the detailed formulas used to express the reliability of any schedule depending on its characteristics (Section 3.4). After a short discussion of the complexity represented by communications in this context (Section 3.5), we conclude in Section 3.6 with an example showing the combinatorial nature of reliability computations. All notations are summarized in Table 1.

\subsection{Application and platform}

The application and platform model is quite simple and borrowed from the scheduling literature (Brucker 2004). The application is represented by a directed acyclic graph (or DAG) $G=(T, E)$, where $T$ is the set of tasks to be executed, and $E$ is the set of dependence edges between the tasks. We let $n=|T|$ be the number of tasks, and we number the tasks $t_{i} \in T, 1 \leq i \leq n$, according to some topological order (which means that if $\left(t_{i}, t_{j}\right) \in E$ then $\left.i<j\right)$. For convenience, we assume that there is a unique source task $t_{1}$ and a unique sink task $t_{n}$. The target platform consists of a set $P$ of $m$ heterogeneous processors $p_{j}, 1 \leq j \leq m$. The execution of task $t_{i}$ on processor $p_{j}$ requires $w_{i}^{j}$ time-units. Note that it is often assumed that $w_{i}^{j}=c_{i} \times \tau_{j}$, where $c_{i}$ is the cost of task $t_{i}$ and $\tau_{j}$ is the cycle-time of processor $p_{j}$ (we then speak of uniform machines). We do not enforce this restriction here, and deal with arbitrary execution times. But without loss of generality, we assume that all execution times $w_{i}^{j}$ are integers, so that time-steps are natural numbers (we can always scale rational values).

\subsection{Failure models}

Processors are subject to failures during the execution of the tasks that are assigned to them. There are two main categories of failures which may occur during the execution of a task $t$ on a processor $p$.

Transient failures: a transient failure will cause the execution of $t$ to fail, but processor $p$ will be available to execute other tasks (the next tasks assigned to it by the scheduler, if any). In other words, $p$ will be able to contribute to the rest of the execution after the transient failure.
Fail-stop failures: a fail-stop failure is an unrecoverable failure that causes the processor to be down until the end of the execution of the whole application: all subsequent tasks assigned to it will not be executed.

Each failure category applies to well-identified scenarios. Transient failures correspond to arithmetic/software errors or recoverable hardware faults (power loss) (Shatz and Wang 1989; Zhu et al 2004). Fail-stop failures correspond to hardware resource crashes, or to the recovery of a loaned machine by her/his user during a cycle-stealing episode (Awerbuch et al 1996; Bhatt et al 1997; Rosenberg 2002).

All our results apply for general distributions, where the failure probabilities are arbitrary rational numbers.

The probability of any fail-stop failure occurring during processor idle times can be transferred to the failure probability of the next scheduled task without modifying the reliability of the schedules. The same idea can be applied if we consider specific transient failures that are undetected when the processor is idle until the next task starts its execution (whose execution would then be unsuccessful). Therefore, without loss of generality, we consider an equivalent model where no failure is assumed to happen during processor idle times.

Finally, processor failures, either transient or fail-stop, are always supposed to be independent, regardless of the distribution laws that they follow.

\subsection{Schedules with task replication}

The objective is to execute the application onto the platform defined above. The schedule assigns tasks to processors. Without replication, each task is assigned to a single processor, with the schedule defining the start-up and completion times of each task onto its assigned processor. However, to remedy the effect of failures, the scheduler may replicate the execution of the tasks onto different processors: if one task fails on a given processor, it is hoped that it will execute successfully on another processor, thereby enabling the rest of the application to proceed despite the failure.

A schedule is thus defined as a one-to-many function which maps each task onto a subset of processors, each of them executing one replica of the task. For each replica, we record a triple of values: the processor number, the start-up time and the failure probability. Formally, $\pi: T \rightarrow 2^{P \times \mathbb{N} \times[0,1]}$ maps every task on a set of such triples. Let $t_{i}^{j}$ be the replica of task $t_{i}$ on processor $p_{j}$ (if it exists). Its start-up time is $S_{i}^{j}$, and its completion time is $C_{i}^{j}=S_{i}^{j}+w_{i}^{j}$. By convention, if $t_{i}$ is not assigned to $p_{j}$, we let $C_{i}^{j}=0$ (and leave $S_{i}^{j}$ undefined). Also, without loss of generality, it is not authorized to schedule twice the same task onto a given processor. This restriction has no impact on our results (scheduling a task 


\begin{tabular}{|c|c|}
\hline Notation & Definition \\
\hline$T=\left\{t_{i}: i \in[1 . . n]\right\}$ & set of tasks \\
\hline$n$ & number of tasks \\
\hline$G=(T, E)$ & directed acyclic graph with tasks and precedence constraints \\
\hline $\operatorname{Pred}\left(t_{i}\right)$ & set of direct predecessors of task $t_{i}$ \\
\hline$P=\left\{p_{j}: j \in[1 . . m]\right\}$ & set of processors \\
\hline & number of processors \\
\hline$\pi: T \rightarrow 2^{P \times \mathbb{N} \times[0,1]}$ & schedule defining the processors, start-up times and failure probabilities of each task \\
\hline$t_{i}^{j}$ & replica of task $t_{i}$ assigned to processor $p_{j}$ \\
\hline$S_{i}^{j}$ & start-up time of $t_{i}^{j}$ (undefined if not scheduled) \\
\hline$w_{i}^{j}$ & execution time of $t_{i}^{j}$ \\
\hline$C_{i}^{j}$ & completion time of $t_{i}^{j}$ ( 0 if not scheduled) \\
\hline $\begin{array}{r}C_{\max }(\pi)=\max _{j} C_{n}^{j} \\
\operatorname{rel}(\pi)\end{array}$ & $\begin{array}{l}\text { makespan of schedule } \pi \\
\text { reliability of schedule } \pi\end{array}$ \\
\hline
\end{tabular}

Table 1: List of notations.

twice on the same processor is at least as hard) but simplifies the notations (e.g., for the completion times $C_{i}^{j}$ ).

The schedule must enforce dependence constraints. Without replication, there is no choice: if there is a dependence from task $t_{i}$ to task $t_{i^{\prime}}$, i.e., if $\left(t_{i}, t_{i^{\prime}}\right) \in E$, then the schedule must enforce that $t_{i^{\prime}}$ cannot start before $t_{i}$ completes: $C_{i}^{j} \leq S_{i^{\prime}}^{j^{\prime}}$, where $t_{i}$ is assigned to $p_{j}$, and $t_{i^{\prime}}$ is assigned to $p_{j^{\prime}}$. When several copies of the same task are executed, there are two possible rules.

Strict schedule: a task cannot start before all copies of each predecessor have completed.

General schedule: a task can start as soon as one replica of each predecessor has completed.

Obviously, strict schedules are a particular case of general schedules. Although they are less general, they are simpler to analyze, at least for transient failures (see Section 5).

It is important to point out that the above definitions apply to a failure-free execution. The start-up and completion times of all tasks are deterministic and known in advance from the schedule definition, before the execution starts. Failures may happen randomly during the execution. See the possible execution with a general schedule on the example in Fig. 1: $t_{2}^{2}$, the replica of $t_{2}$ on $p_{2}$, can start as soon as $t_{1}^{1}$, the replica of $t_{1}$ on $p_{1}$, has completed (there is no need to wait for the completion of the other replica $t_{1}^{3}$ of $t_{1}$ on $p_{3}$ ). However, if $t_{1}^{1}$ fails during execution, then $t_{2}^{2}$ becomes useless.

Now, for each dependence edge $\left(t_{i}, t_{i^{\prime}}\right) \in E$ and for each processor pair $\left(p_{j}, p_{j^{\prime}}\right) \in P^{2}$, there are two cases: if the completion time $C_{i}^{j}$ of the replica $t_{i}^{j}$ of $t_{i}$ is not larger than the start-up time $S_{i^{\prime}}^{j^{\prime}}$ of the replica $t_{i^{\prime}}^{j^{\prime}}$ of $t_{i^{\prime}}$, we say that the replica pair $\left(t_{i}^{j}, t_{i^{\prime}}^{j^{\prime}}\right)$ is valid; otherwise, we say that it is not valid.

For a strict schedule, all replica pairs must be valid for every precedence edge in the task graph. For a general schedule, this constraint is not enforced. However, for each path in the task graph, there must be a list of replicas that correspond to the tasks of the path and such that each replica forms valid replica pairs with its neighbors in the list: if it is not the case, the schedule will never be able to complete its execution, even without any failure. Intuitively, we expect strict schedules to be more reliable than general schedules:

- for a strict schedule, a task will be able to start execution if and only if at least one replica of each of its predecessors has been successfully executed,

- while for a general schedule, the range of admissible predecessor copies is restricted to those whose completion time is not later than the task start-up time.

However, the total execution time, or makespan, is likely to be smaller for general schedules than for strict schedules, because there are fewer replica pairs that are accounted for, hence fewer predecessor copies to wait for. Recall that the makespan $C_{\max }(\pi)$ of a schedule $\pi$ is formally defined as the completion time of the last replica of the sink task $t_{n}$.

The proposed scheduling mechanism is static: no adjustment is done during the execution, relatively to the replicas that succeed and the failures that occur. As such, failures do not require to be detected (the execution of the schedule is pursued until the end). Although dynamic approaches are more practical in presence of high uncertainty, pro-actively evaluating the reliability of the scheduling decisions is still required. Therefore, static and dynamic approaches are complementary and raise a similar evaluation problem.

\subsection{Reliability}

Similarly to Barlow and Proschan (1967), we consider the execution of the schedule until its first failure. As stated previously, the failure of one replica may not cause the schedule to fail. The reliability $\operatorname{rel}(\pi)$ of a schedule $\pi$ is defined as the probability that the schedule is successful, i.e., succeeds to complete its execution. A strict schedule is easily checked to be successful if and only if at least one replica of each task completes its execution. Determining whether a general schedule is successful is more complicated: we traverse 
the DAG to check whether the execution of each replica is successful or not. More precisely, a replica $t_{i^{\prime}}^{j^{\prime}}$ of a task $t_{i^{\prime}}$ is successful if and only if:

1. $p_{j^{\prime}}$ does not fail during the execution of $t_{i^{\prime}}^{j^{\prime}}$, and

2. for each predecessor $t_{i} \in \operatorname{Pred}\left(t_{i^{\prime}}\right)$ (if any), there exists at least one valid replica pair $\left(t_{i}^{j}, t_{i^{\prime}}^{j^{\prime}}\right)$ such that $t_{i}^{j}$ is successful.

Finally, a general schedule is successful if at least one replica of the sink task $t_{n}$ is successful (because it induces that each task has been successfully computed at least once).

In order to formally define the reliability, we use several events that denote each a set of outcomes of the sample space. Usual notations of set theory are used to represent disjunction and conjunction (union and intersection, respectively). The following definitions are based on two types of events, which enable a formal and complete proof of our completeness result.

Let $\pi$ be a schedule. Then

- $R_{i j}$ denotes the event that processor $p_{j}$ does not fail during the execution of $t_{i}^{j}$, and $\operatorname{Pr}\left[R_{i j}\right]$ denotes the probability of this event. With transient failures, this simply means that $p_{j}$ does not fail between the start-up and completion times of $t_{i}^{j}$, while with fail-stop failures this means that $p_{j}$ does not fail from the beginning of the schedule until the end of execution of $t_{i}^{j}$. Note that this event is necessary but not sufficient for replica $t_{i}^{j}$ to be successful: a valid replica of each predecessor of $t_{i}$ must have been successfully executed too. Let $\operatorname{Pr}\left[R_{i j}\right]=0$ if task $t_{i}$ is not assigned to processor $p_{j}$.

- $U_{i j}$ denotes the event that replica $t_{i}^{j}$ of task $t_{i}$ is successful, and $\operatorname{Pr}\left[U_{i j}\right]$ denotes the probability of this event. Let $\operatorname{Pr}\left[U_{i j}\right]=0$ if task $t_{i}$ is not assigned to processor $p_{j}$. Otherwise, event $U_{i j}$ is defined as follows:

$$
U_{i j}=\left\{\left(\begin{array}{ll}
R_{i j} & \text { if } \operatorname{Pred}(i)= \\
\bigcap_{i^{\prime} \in \operatorname{Pred}(i)} \bigcup_{j^{\prime}, C_{i^{\prime}}^{j^{\prime}} \leq S_{i}^{j}} U_{i^{\prime} j^{\prime}}
\end{array}\right) \cap R_{i j}\right. \text { otherwise }
$$

Note that the initialisation only applies to $i=1$, as $t_{1}$ is a unique source task. Note also that the set of predecessor copies has been restrained to valid replica pairs, i.e., to any predecessor copy $t_{i^{\prime}}^{j^{\prime}}$ such that $C_{i^{\prime}}^{j^{\prime}} \leq S_{i}^{j}$.

- The reliability $\operatorname{rel}(\pi)$ of a general schedule $\pi$ is the probability that at least one replica of the sink task is successful:

$$
\operatorname{rel}(\pi)=\operatorname{Pr}\left[\bigcup_{j} U_{n j}\right] .
$$

- The reliability $\operatorname{rel}(\pi)$ of a strict schedule $\pi$ is the probability that at least one replica of each task is successful and is defined as

$\operatorname{rel}(\pi)=\operatorname{Pr}\left[\bigcap_{i} \bigcup_{j} R_{i j}\right]$.

Note that the $U_{i j}$ are not needed to compute the reliability for strict schedules because all replica pairs are valid.

Finally, we assume for simplicity that the schedule is non-preemptive, but the proof of Theorem 1 shows that the \#P'-Completeness result holds true for preemptive schedules.

\subsection{Communications}

We point out that edge failures and communication costs could easily be taken into account when evaluating the reliability of a schedule: replace each dependence edge $t_{i}^{j} \rightarrow t_{i^{\prime}}^{j^{\prime}}$ by two edges $t_{i}^{j} \rightarrow \operatorname{comm}_{i i^{\prime}}^{j j^{\prime}} \rightarrow t_{i^{\prime}}^{j^{\prime}}$, where comm $i i^{\prime} j^{\prime}$ is a new task whose execution time is the communication cost between the two replicas, and whose failure probability (either transient or fail-stop) can be freely chosen. In the case of fail-stop failures, each task comm ${ }_{i i^{\prime}}^{j j^{\prime}}$ must be scheduled on a processor of its own. The edge failure probability is likely to depend upon the communication cost and/or the link failure rate. If $j=j^{\prime}$, we model failures during memory transfer; otherwise, we model failures across interconnection links. Altogether, we can deal with communications by adding $|E|$ tasks and processors (where $E$ is the set of edges).

\subsection{Example}

In this section, we deal with a toy example showing the difficulty of computing the reliability in the presence of fail-stop failures, even with independent tasks. Note that all schedules are both strict and general in the case of independent tasks, since there are no dependence constraints. Fig. 3 illustrates a schedule with two tasks and four processors, which all execute both tasks (but in different orders). Each task is thus replicated four times. For each processor $p_{j}, P_{1 j}$ denotes the probability that $p_{j}$ fails during the execution of its first replica; $P_{2 j}$ denotes the probability that $p_{j}$ fails during the execution of its second replica; and $P_{3 j}$ denotes the probability that $p_{j}$ does not fail before the completion of both replicas. The direct approach to evaluate the schedule reliability consists in forming all the products $P_{a 1} P_{b 2} P_{c 3} P_{d 4}$ with $a, b, c, d \in$ $\{1,2,3\}$. Each product is the probability that a specific execution scenario occurs, and all these scenarios are distinct. Therefore, we can add the terms corresponding to successful scenarios for computing the reliability of the schedule. For instance, $P_{11} P_{22} P_{23} P_{14}$ is the probability that $p_{1}$ and $p_{2}$ fail while computing their replicas of $t_{1}$, and $p_{3}$ and $p_{4}$ fail while 


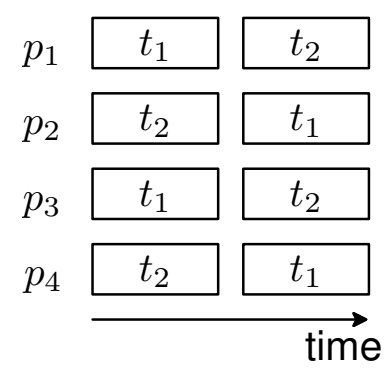

\begin{tabular}{|c|c|}
\cline { 2 - 2 } \multicolumn{1}{c|}{} & Reliability (rel $(\pi))$ \\
\hline$p_{1}$ & $P_{31}$ \\
\hline$p_{1}, p_{2}$ & $P_{11} P_{32}+P_{21}\left(P_{22}+P_{32}\right)+P_{31}$ \\
\hline$p_{1}, p_{2}, p_{3}$ & $P_{11}\left(P_{12} P_{33}+P_{22}\left(P_{23}+P_{33}\right)+P_{32}\right)+P_{21}\left(P_{12} P_{33}+P_{22}+\right.$ \\
& $\left.P_{32}\right)+P_{31}$ \\
\hline & $P_{11}\left(P_{12}\left(P_{13} P_{34}+P_{23}\left(P_{24}+P_{34}\right)+P_{33}\right)+P_{22}\left(P_{13} P_{34}+\right.\right.$ \\
$p_{1}, p_{2}, p_{3}, p_{4}$ & $\left.\left.P_{23}+P_{33}\right)+P_{32}\right)+P_{21}\left(P_{12}\left(\left(P_{13}+P_{23}\right)\left(P_{24}+P_{34}\right)+\right.\right.$ \\
& $\left.\left.P_{33}\right)+P_{22}+P_{32}\right)+P_{31}$ \\
\hline
\end{tabular}

Fig. 3: Schedule with two independent tasks on four processors.

computing their replicas of $t_{2}$. This scenario is actually successful as $t_{2}$ is computed by $p_{2}$ and $t_{1}$ by $p_{3}$. The table in Fig. 3 shows the formulas obtained with this approach. Each formula defines the reliability when only the subset of processors defined in the first column is used. We remark that the number of terms grows exponentially with the number of processors, and that it does not seem possible to factor the terms into a compact formula.

\section{Complexity of general schedules}

In this section, we show that computing the reliability of a general schedule is a \#P'-Complete problem. This holds both for transient and failure-stop failures, and for arbitrary rational failure probabilities (we cannot deal with real numbers when assessing problem complexity). We start with a definition of the \#P' complexity class and formally state the problem before providing a fully detailed proof, which we decompose into several steps.

\subsection{Problem statement}

Informally, Valiant (1979) introduced the notions of \#P and \#P-Completeness to express the hardness of problems that "count the number of solutions". Because counting a number of solutions to a problem is at least as hard as determining if there is at least a solution, \#P problems are at least as difficult as their corresponding NP problems. There is a technical complication with schedule reliability problems, just as with network reliability problems (Provan and Ball 1983; Bodlaender and Wolle 2004): we are dealing with probability values, which are rational numbers in $[0,1]$, instead of dealing with integers as in Valiant (1979). Thus, we follow Bodlaender and Wolle (2004) and establish the \#P'-Completeness of the problem. The \#P' class is a natural extension of the class \#P to deal with rational numbers: it allows us to apply a polynomial function on the \#P integer output, producing in our case a rational number. The formal definitions are the following:
Definition 1 (Complexity classes) Let $\Sigma$ be a finite alphabet and $\Sigma^{*}$ be the set of all strings over $\Sigma$.

- The class \#P consists of the functions $f: \Sigma^{*} \Rightarrow \mathbb{N}$ such that there exists a nondeterministic polynomial-time Turing machine $M$ such that for all inputs $x \in \Sigma^{*}, f(x)$ is the number of accepting paths of $M$.

- The class \#P' consists of the functions $h: \Sigma^{*} \Rightarrow \Sigma^{*}$ such that there exists a function $f \in \# P, f: \Sigma^{*} \Rightarrow \mathbb{N}$, and a polynomial-time computable function $g: \mathbb{N} \times \Sigma^{*} \Rightarrow \Sigma^{*}$, which satisfy to $\forall x \in \Sigma^{*}, h(x)=g(f(x), x)$.

Definition 2 (SCHEDULE-RELIABILITY) Given a general schedule $\pi$, SCHEDULE-RELIABILITY is the problem of computing the reliability $\operatorname{rel}(\pi)$ as defined by Equations (1) and (2), for arbitrary rational failure probabilities $\operatorname{Pr}\left[R_{i j}\right]$.

We are ready to state the main result:

\section{Theorem 1 Schedule-Reliability is \#P'-Complete.}

The motivation for introducing the class \#P' should be clearer now: the problem is to compute the rational value $\operatorname{rel}(\pi)$ for a general schedule $\pi$, rather than the number of successful executions of $\pi$ for all possible failure scenarios.

The proof of Theorem 1 shows that the result holds for both transient and fail-stop failures. The proof goes just as an NP-Completeness proof: we first prove in Lemma 1 that the problem belongs to the $\# P^{\prime}$ class (SCHEDULE-RELIABILITY $\in \# P^{\prime}$ ), and then we prove its hardness in Lemma 2 by reduction from another \#P'-Complete problem, namely the CONNECTED problem, which we define below:

Definition 3 (CONneCTED) Given a DAG $G=(A, B)$ with a source and sink nodes, and whose edges are subject to failures with rational independent probabilities, CONNECTED is the problem of computing the probability that the source and the sink nodes are joined by a path of non-failing edges.

Connected is \#P'-Complete (Provan and Ball 1983, Problem 10 and Section 3). In fact, it is a slight variant of the two terminal network reliability problem in Provan and Ball (1983): instead of joining two arbitrary nodes of the graph, we join the source and the sink. The reduction from the original problem is straightforward. 

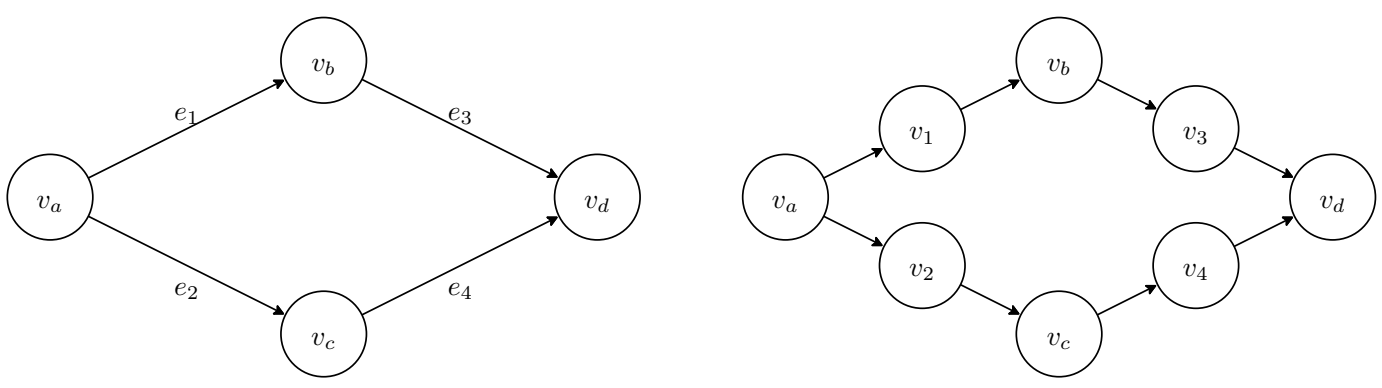

Fig. 4: An instance of CONNECTED and its transformation.

\subsection{Class membership}

\section{Lemma 1 SCHEDUle-Reliability is in \#P'.}

Proof In order to prove that the SCHEDULE-RELIABILITY belongs to \#P', we need to characterize the underlying NP decision problem and the transformation for generating the output probability, which is a rational number.

The success probabilities of each processor $p_{j}$ while computing each task $t_{i}$ are all assumed to be encoded as $\frac{n_{i j}}{d_{i j}}$. A vector $x_{i}=\left(x_{i j}\right)_{1 \leq j \leq m}$ specifies the success of each processor $p_{j}$ when computing task $t_{i}$. If $1 \leq x_{i j} \leq n_{i j}$, then $p_{j}$ does not fail while computing $t_{i}$. If $n_{i j}<x_{i j} \leq d_{i j}$, then $p_{j}$ fails while computing $t_{i}$. Otherwise, $t_{i}$ is not assigned to $p_{j}$ and $x_{i j}=0\left(d_{i j}=1\right.$ and $n_{i j}$ is left undefined in this case $)$.

The NP decision problem is the following: given a schedule, does there exist a vector $x_{i}$ such that the schedule terminates successfully? This problem belongs to NP because the vector $x=\left(x_{i}\right)_{1 \leq i \leq n}$ of size $O(n m)$ constitutes the certificate. We check whether a vector $x$ encodes a successful schedule execution by building a schedule containing only tasks without failures. If such a schedule is valid, namely, if all precedence constraints are respected and if all tasks are correctly computed (see Section 3 for more details on this schedule verification procedure), then $x$ encodes a successful schedule execution.

The corresponding \#P problem consists in computing how many distinct vectors $x$ give successful schedules. In other words, there are $\prod_{i=1}^{n} \prod_{j=1}^{m} d_{i j}$ distinct vectors and each of them defines a possible scenario for the schedule execution. Because all scenarios are equiprobable, we obtain the reliability of a schedule by dividing the number of successful scenarios by the total number of scenarios.

This proves the membership of SCHEDULE-RELIABILITY to the \#P' complexity class.

\subsection{Completeness}

\section{Lemma 2 Schedule-Reliability is \#P'-Hard.}

Proof The proof is rather involved. We start with an arbitrary instance Inst $_{1}$ of CONNECTED and we build an in- stance Inst $_{2}$ of SCHEDULE-RELIABILITY such that the probability that the source and the sink nodes are joined by a path of non-failing edges in Inst $_{1}$ is equal to the unreliability of the general schedule in Inst $_{2}$. For better readability, we divide the reduction into several steps.

Step 1: Transformation of Inst ${ }_{1}$. We first transform the DAG $G=(A, B)$ with a source and sink nodes of Inst $t_{1}$, and provide some formal notations. We move from an edge-failing problem to a vertex-failing problem. These vertices will correspond to scheduled tasks in Inst $_{2}$. Each edge $(i, j)$ in $B$ from vertex $i$ to vertex $j$ is replaced by a new vertex $k$, and by two edges, $(i, k)$ from $i$ to $k$, and $(k, j)$ from $k$ to $j$, as illustrated on Fig. 4: the instance of CONNECTED has four nodes, and the transformed instance has eight nodes. The failure probability of $(i, j)$ is transferred to the new vertex $k$. All original vertices never fail, hence, the probability that the source and the sink are joined is identical to that in the original DAG $G$.

There are $n=|A|+|B|$ vertices in the new DAG, which we number according to a topological ordering (hence, 1 is the source node and $n$ is the sink node). Moreover, any generated graph with this procedure has a special structure, e.g., any vertex has either one successor, or its successors have only one predecessor. Let $N_{i}$ be the event that the vertex $i$ is valid (does not fail). As already mentioned, the success probability of each $|A|$ vertex already present in the original DAG is equal to 1 . Let $V_{i}$ be the event that there is a path between the source node and node $i$. Evaluating the reliability in the CONNECTED problem requires to compute $\operatorname{Pr}\left[V_{n}\right]$, where $V_{1}=N_{1}$ and $V_{i}$ is defined recursively for $i>1$ as

$V_{i}=\bigcup_{i^{\prime} \in \operatorname{Pred}(i)}\left(V_{i^{\prime}} \cap N_{i^{\prime}}\right)$

Step 2: Construction of Inst 2 . A task is created in Inst 2 for each vertex of the transformed version of Inst $t_{1}$. The execution time of each task replica on each processor is equal to 1. Each task is scheduled on a processor with success probability equal to the probability that the corresponding vertex in the CONNECTED instance fails. The success probability of the CONNECTED instance will be shown to be equal to 


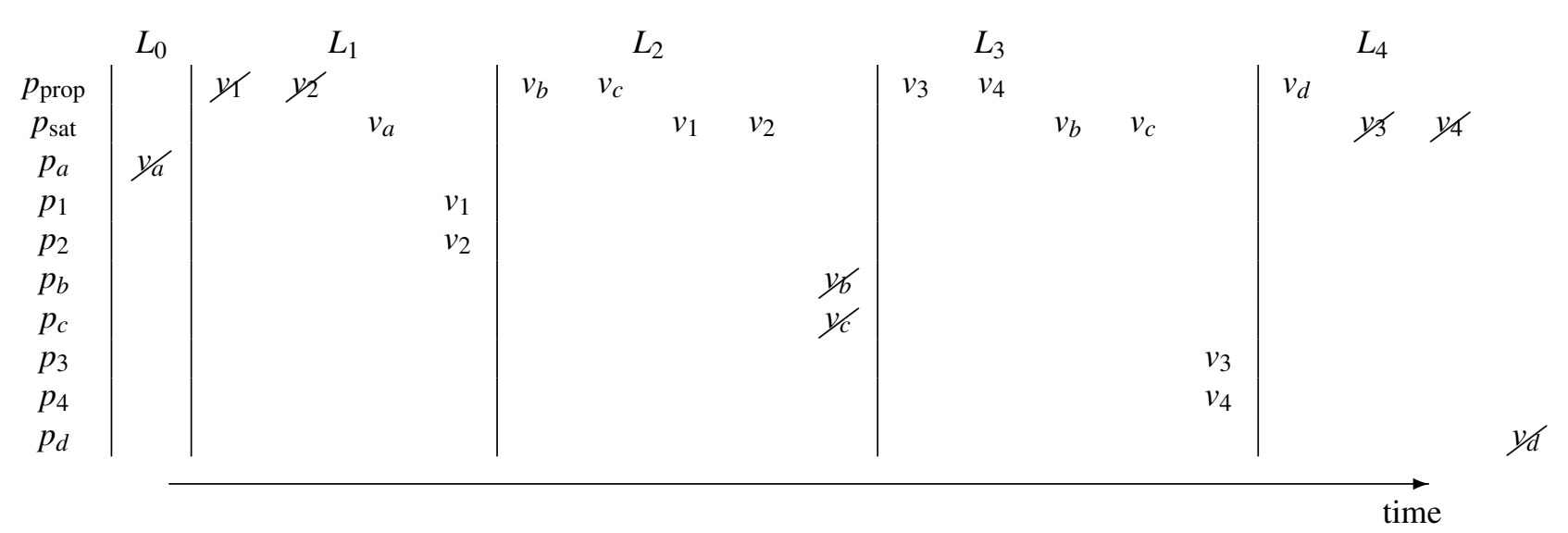

Fig. 5: Schedule built by the reduction algorithm for the instance of Fig. 4. Canceled replicas can be discarded as they do not impact the schedule reliability. It is the case for vertices $v_{a}, v_{b}, v_{c}$ and $v_{d}$ that are scheduled on their specific processors and all have a zero probability of success.

the failure probability of the schedule created by the reduction algorithm (Algorithm 1). In fact, the schedule succeeds (no successful path in CONNECTED) if some subset of tasks succeed on their specific processors (some subset of vertices fail in CONNECTED). If the schedule is globally successful, then no path is successful in the CONNECTED instance.

The reduction algorithm starts by grouping vertices into several levels through a breadth-first search. All the vertices at depth $i$ are put in the $i$-th level. Then, a task is created for each vertex of CONNECTED and is scheduled three times, except for the sink and source vertices which have only two replicas.

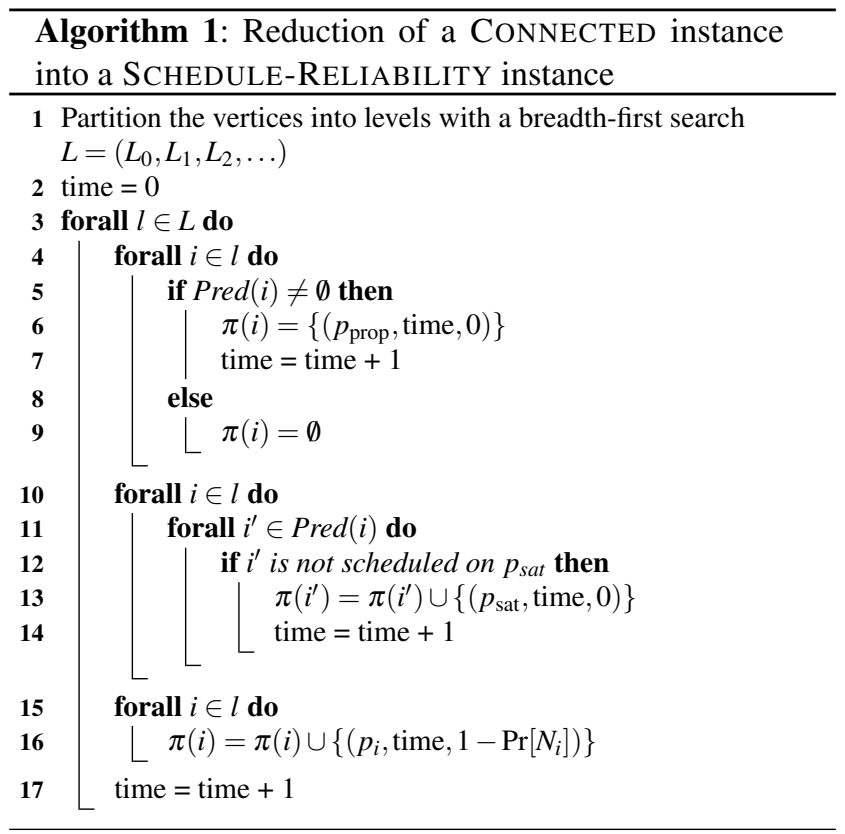

The propagate processor, $p_{\text {prop }}$, and the satisfy processor, $p_{\text {sat }}$, play a special role and execute all tasks except the source and sink. These processors never fail. Each task is also scheduled on a specific processor whose index is the same as the task index (i.e., task $t_{i}$ is mapped on processor $p_{i}$ ). Intuitively, the role of processor $p_{\text {prop }}$ is to "propagate" the success of one task to its successors. This notion of "propagation" is best understood in the CONNECTED instance: one vertex might be successful, yet unreachable, in which case the failure of its ancestors must be "propagated" to it. Keeping track of failures (successes in the schedule) is mandatory for the reduction to be effective. Initially, the precedence constraints for the replicas on $p_{\text {prop }}$ cannot be satisfied by the replicas scheduled on the processors $p_{\text {prop }}$ or $p_{\text {sat }}$. But anytime a task $t$ succeeds on its specific processor, the precedence constraints between $t$ and its successors scheduled on $p_{\text {prop }}$ are satisfied. If all the precedence constraints of these successors are satisfied, then they are suc-

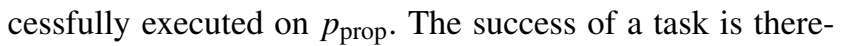
fore "propagated" to its successors, which may succeed even if their replicas scheduled on their specific processors fail. Here, the key idea lies in the fact that each task scheduled on its specific processor finishes before that any of its successors scheduled on $p_{\text {prop }}$ starts. Moreover, with processor $p_{\text {sat }}$, the precedence constraints of each task scheduled on its specific processor are satisfied. Indeed, we want tasks scheduled on their specific processors to succeed independently of their precedence constraints. Therefore, all the ancestors of a task $t_{i}$ must succeed before time $S_{i}^{i}$. Processor $p_{\text {sat }}$ plays this role by successfully computing each task in a topological order. Note that two distinct fully reliable processors are used because the model forbids to schedule the same task twice on the same processor.

In the example of Fig. 5, the breadth-first search generates five levels: $\left\{v_{a}\right\},\left\{v_{1}, v_{2}\right\},\left\{v_{b}, v_{c}\right\},\left\{v_{3}, v_{4}\right\}$ and $\left\{v_{d}\right\}$. 
All the successors of the predecessors of one level are in the same level. For level $L_{3}$, the three steps of the main loop of the reduction algorithm consist in: scheduling the tasks of the level, $v_{3}$ and $v_{4}$, on $p_{\text {prop }}$; scheduling the predecessors of these tasks, $v_{b}$ and $v_{c}$, on $p_{\text {sat }}$; and scheduling the tasks in $L_{3}$ to their specific processors, $p_{3}$ and $p_{4}$. If $v_{1}$ is successful on $p_{1}$, then $v_{3}$ is successful on $p_{\text {prop }}$, which shows the "propagation" of task successes (corresponding to edge failures in the CONNECTED instance). Otherwise, $v_{3}$ can still be successfully on $p_{3}$. In both cases, the schedule is successful if $v_{d}$ succeeds on $p_{\text {prop }}$, i.e., if both $v_{3}$ and $v_{4}$ are successful. In the CONNECTED instance, it is equivalent to state that there is no path from the source to $v_{d}$ if there is no path neither to $v_{3}$ nor to $v_{4}$.

Step 3: Equivalence of Inst $t_{1}$ and Inst In $_{2}$ We now show that the success probability of Inst $t_{1}$ is equal to the failure probability of Inst $t_{2}$. The roadmap is the following. We first propose in Lemma 3 a simplification of the recursive equation (1) defining the reliability of a general schedule, which shows that the success of a task on $p_{\text {prop }}$ depends on the successes of all its predecessors, either because they succeed on their specific processor or because their replica on $p_{\text {prop }}$ is successful. This means that the success of a task (the failure of a path) is "propagated" to its successors.

Then, we introduce the correspondence between the failure events of the CONNECTED and SCHEDULE-RELIABILITY instances: we show that any task scheduled on its specific processor has all its precedence constraints satisfied due to the replicas scheduled on $p_{\text {sat }}$ (see Lemma 4).

Building upon these two lemmas, we can then prove the equivalence of solutions by showing that

$$
V_{n}=\overline{\bigcup_{j} U_{n j}}=\overline{U_{n \text { prop }}}
$$

Notations concerning the events that will be manipulated are summarized in Table 2.

\section{Step 3.1: Simplifying probabilities.}

Lemma 3 Consider the schedule of Inst $t_{2}$. Then, the success of any task $t_{i} \in T$ on processor $p_{\text {prop }}$ is given by $U_{\text {iprop }}=$ $\bigcap_{i^{\prime} \in \operatorname{Pred}(i)}\left(U_{i^{\prime} \text { prop }} \cup U_{i^{\prime} i^{\prime}}\right)$.

Proof Using Equation (1), we obtain

$U_{\text {iprop }}=\left(\bigcap_{i^{\prime} \in \operatorname{Pred}(i)} \bigcup_{j^{\prime}, C_{i^{\prime}}^{j^{\prime}} \leq S_{i}^{\text {prop }}} U_{i^{\prime} j^{\prime}}\right) \cap R_{i \text { prop }}$.

By construction, tasks never fail on $p_{\text {prop }}$ or $p_{\text {sat }}$ processors. Thus, $R_{\text {iprop }}$ occurs almost surely (with probability 1 ) and this term can be discarded. We further simplify by expanding the internal union. Each predecessor $t_{i^{\prime}}$ of a task $t_{i}$ is scheduled three times: on processor $p_{\text {prop }}$, except for the source; on processor $p_{\text {sat }}$, except for the sink; and on its specific processor. We now characterize which replicas $t_{i^{\prime}}^{j}$ of the predecessor $t_{i^{\prime}}$ are completed before $t_{i}$ starts on $p_{\text {prop }}$, i.e., $C_{i^{\prime}}^{j} \leq S_{i}^{\text {prop }}$.

Any task $t \in T$ (except the source) in the $k$-th level is scheduled on $p_{\text {prop }}$ and on its specific processor at the $k$-th iteration. Thus, when any successor of $t$ in the $k^{\prime}$-th level, with $k^{\prime}>k$, is scheduled on $p_{\text {prop }}$ at the $k^{\prime}$-th iteration, $t$ has already been finished on $p_{\text {prop }}$ and on its specific processor. Formally, if $t_{i^{\prime}}$ is a predecessor of $t_{i}$, then $C_{i^{\prime}}^{\text {prop }} \leq S_{i}^{\text {prop }}$ and $C_{i^{\prime}}^{i^{\prime}} \leq S_{i}^{\text {prop }}$.

We now show by contradiction that any task finishes its execution on $p_{\text {sat }}$ after that any of its successors starts on $p_{\text {prop }}$ (i.e., $C_{i^{\prime}}^{\mathrm{sat}}>S_{i}^{\mathrm{prop}}$ ). This allows the expansion of the internal union without considering the success of predecessors scheduled on $p_{\text {sat }}$.

In Algorithm 1, consider a task $t \in T$ whose depth is $k$. If $t$ is not the source, then $t$ is scheduled on $p_{\text {prop }}$ (on Line 5) at the $k$-th iteration because the breadth-first search puts $t$ in the $k$-th level. Moreover, $t$ is scheduled on $p_{\text {sat }}$ (on Line 12) after the $k$-th iteration because all the successors of $t$ are in the following levels. Now, suppose that $t$ finishes on $p_{\text {sat }}$ before that one of its successors $t^{\prime}$ in the $k^{\prime}$-th level, with $k^{\prime}>k$, start on $p_{\text {prop. }}$. Then, $t$ is scheduled on $p_{\text {sat }}$ before that $t^{\prime}$ is scheduled on $p_{\text {prop }}$ because task costs and time increments are all unitary. At the $k^{\prime}$-th iteration, $t^{\prime}$ is scheduled on $p_{\text {prop }}$ before any task is scheduled on $p_{\text {sat }}$. Therefore, $t$ must have another successor whose depth is lower than $k^{\prime}$, otherwise $t$ would not be scheduled on $p_{\text {sat }}$ before the $k^{\prime}$-th iteration. It implies that $k^{\prime}>k+1$, i.e., there is one level that contains this other successor between the $k$-th and the $k^{\prime}$-th levels. Thus, $t^{\prime}$ has a predecessor in the $k^{\prime}-1$-th level because the depth of $t^{\prime}$ is $k^{\prime}$. This predecessor cannot be $t$ because $t$ is in the $k$-th level and $k<k^{\prime}-1$. We see that $t$ has two successors, among which $t^{\prime}$, which has also two predecessors. There are two cases: either $t$ corresponds to a vertex in the original DAG of the CONNECTED instance, or it corresponds to an edge transformed into a vertex. In the first case, it means that $t^{\prime}$ corresponds to an edge. However, the vertices resulting from the edges have only one predecessor, which contradicts the fact that $t^{\prime}$ has at least two ones. In the second case, $t$ comes from an edge. But then, it should have a single successor, instead of two ones. Therefore, there is no task that finishes on $p_{\text {sat }}$ before that one of its successor starts on $p_{\text {prop. }}$.

\section{Step 3.2: Correspondence between failure events}

Lemma 4 Consider the schedule of Inst I $_{2}$ For any task $t_{i} \in$ $T$, assume that its specific processor succeeds during its execution $\left(R_{i i}\right)$ whenever its corresponding vertex in the $\mathrm{CON}$ NECTED instance fails $\left(\overline{N_{i}}\right)$, and reciprocally. Then, each 


\begin{tabular}{r|l}
\hline Symbol & Definition \\
\hline$R_{i j}$ & event that processor $p_{j}$ does not fail before the end time of replica $t_{i}^{j}$ \\
$U_{i j}$ & event that replica $t_{i}^{j}$ is successfully processed \\
$N_{i}$ & event that the node $i$ is valid (for CONNECTED) \\
$V_{i}$ & event that a path exists between the source and node $i$ (for CONNECTED) \\
$\operatorname{Pr}[X]$ & probability of event $X$ \\
\hline
\end{tabular}

Table 2: List of notations for Lemmas 3 and 4.

task $t_{i}$ succeeds on its specific processor if and only if its corresponding vertex in the CONNECTED instance fails, i.e., $U_{i i}=\overline{N_{i}}$.

Proof We first prove that all the ancestors of task $t_{i}$ are scheduled on processor $p_{\text {sat }}$ in a topological order. More precisely, we show by induction on the levels, that each task of the first $k$ levels starts on its specific processor after that all its ancestors have been scheduled in a topological order on $p_{\text {sat }}$. The basis for the induction is easily verified for $k=0$. Indeed, the source task does not have any ancestor, therefore it is true. Now, assume the induction hypothesis to be true for a given $k$. Let $t$ be a task in the $(k+1)$-th level. At the $(k+1)$-th iteration, $t$ is scheduled on its specific processor (on Line 15) after its predecessors are scheduled on $p_{\text {sat }}$ (on Line 12). These predecessors belong to the first $k$ levels. Thus, their ancestors are scheduled in a topological order on $p_{\text {sat }}$ during the first $k$ iterations (by induction hypothesis). As task costs and time increments are unitary, tasks scheduled at the $(k+1)$-th iteration start after that all earlier scheduled tasks have finished. Therefore, the ancestors of the predecessors of $t$ are scheduled in a topological order on $p_{\text {sat }}$ and the predecessors of $t$ that are not yet scheduled on $p_{\text {sat }}$ are scheduled on it in an arbitrary order at the $k$-th iteration. As these predecessors of $t$ belongs to the same level, they have the same depth and do not require to be scheduled in any specific order for their precedence constraints to be satisfied. Hence, $t$ starts on its specific processor after all its ancestors have finished on $p_{\text {sat }}$.

As a consequence, all the ancestors of task $t_{i}$ are scheduled on $p_{\text {sat }}$ and finish before that $t_{i}$ starts on its specific processor, $p_{i}$. Additionally, the ancestors of $t_{i}$ succeed with probability 1 because tasks scheduled on $p_{\text {sat }}$ never fail (see Line 12). Thus, when $t_{i}$ starts its execution on $p_{i}$, all its precedence constraints are almost surely satisfied. Moreover, there is no other task scheduled on $p_{i}$. Therefore, the success of $t_{i}$ depends only on its execution, i.e., $U_{i i}=R_{i i}=\overline{N_{i}}$.

Step 3.3: Equivalence of both instances. We now show that the success probability of Inst $_{1}$ is equal to the failure probability of Inst $t_{2}$. More precisely, we prove that $V_{n}=\overline{U_{j} U_{n j}}=$ $\overline{U_{n \text { prop }}}$ (recall that task $t_{n}$ fails almost surely on its specific processor, and that it is not scheduled on processor $\left.p_{\text {sat }}\right)$.
The relation between the definitions of $V_{i}$ (Equation (4)) and $U_{i j}$ (Equation (1) in Section 3) is obtained using Morgan's law $\overline{X \cup Y}=\bar{X} \cap \bar{Y}$ and Lemmas 3 and 4 .

Without loss of generality, assume that tasks are sorted in a topological order. We proceed by induction and show that for each task $t_{i}$, the success of vertex $i$ is equivalent to the failure of $t_{i}$ on processor $p_{\text {prop }}$, i.e., $\forall i, V_{i}=\overline{U_{i \text { prop }}}$.

For $i=1$, the source node is not scheduled on processor $p_{\text {prop }}$ because it does not have any predecessor. Hence, $U_{i \text { prop }}$ never occurs. On the other hand, the source vertex is present in the original CONNECTED instance and always succeeds, implying that $V_{i}$ always occurs. Therefore, the basis of the induction is verified, i.e., $V_{1}=\overline{U_{1 \text { prop }}}$.

For a task $t_{i}$, we suppose that $V_{k}=\overline{U_{k p r o p}}$ is true for $1 \leq$ $k<i$. Let us show that $V_{i}=\overline{U_{i p r o p}}$ is also true.

$$
\begin{aligned}
& V_{i}=\bigcup_{i^{\prime} \in \operatorname{Pred}(i)} V_{i^{\prime}} \cap N_{i^{\prime}} \quad \text { by Equation (4) } \\
& =\bigcup_{i^{\prime} \in \operatorname{Pred}(i)} \overline{U_{i^{\prime} \text { prop }}} \cap N_{i^{\prime}} \text { by induction hypothesis } \\
& =\bigcup_{i^{\prime} \in \operatorname{Pred}(i)} \overline{U_{i^{\prime} \text { prop }}} \cap \overline{U_{i^{\prime} i^{\prime}}} \text { by Lemma } 4 \\
& =\overline{\bigcap_{i^{\prime} \in \operatorname{Pred}(i)} U_{i^{\prime} \operatorname{prop}} \cup U_{i^{\prime} i^{\prime}}} \text { by Morgan's Law } \\
& =\overline{U_{\text {iprop }}} \quad \text { by Lemma } 3
\end{aligned}
$$

In the second line, we have used that $i^{\prime}<i$ because tasks are traversed in a topological order. In the third line, the assumption of Lemma 4 holds by construction: all events $N_{i}$ are independent, all events $R_{i j}$ are indeed independent, and the probabilities are identical, i.e., $\operatorname{Pr}\left[R_{i i}\right]=1-\operatorname{Pr}\left[N_{i}\right]$ for all $i$.

We have shown that the reduction algorithm is correct. Assessing its space polynomial complexity is done by counting the number of processors used, the number of replicas scheduled and the space required to store the probabilities. The algorithm schedules each of the $n=|A|+|B|$ tasks at most three times on $n+2$ processors. Probabilities are computed and stored through a basic arithmetic operation $(y \leftarrow 1-x)$. For the time complexity, the number of calls to Lines 5 and 15 are linear in $n$. Finally, using an adequate data structure, the condition on Line 11 can be checked in constant time, and Line 12 is called a number of times linear in $n$. This concludes the proof.

As already mentioned, the proof of Theorem 1 does not depend upon whether failures are transient or fail-stop. Hence, Theorem 1 is valid for any general schedule. Also, failure probabilities can be arbitrary rational numbers. As the proof 
only requires processors to be identical and tasks to have unitary costs, the complexity of the problem is related to the DAG structure only. Altogether, the previous complexity result is relevant to quite a wide class of DAG scheduling problems with replication. For instance the result is also valid for preemptive schedules: interrupting the execution of a task may modify the probability of failure of that task, but the proof handles arbitrary failure probabilities.

Finally, the reduction proof shows that evaluating the reliability of any CONNECTED instance exactly amounts to evaluating the unreliability of the schedule generated by the reduction. We deduce from (Provan and Ball 1983, Problem 10 and Section 3 ) the following corollary:

Corollary 1 Approximating the reliability of a general schedule up to an arbitrary quantity $\varepsilon$ or to an arbitrary ratio $\alpha$ is \#P'-Complete.

\section{Complexity of strict schedules}

For transient failures, a closed-form formula for computing the reliability is provided in Girault et al (2009). This formula can be evaluated in polynomial time, and it can be further simplified in case of Poisson processes.

We focus now on fail-stop failures. While the case without replication still has polynomial-time complexity, the case with replication is open (to the best of our knowledge). We conjecture that evaluating the reliability of strict schedules has the same complexity as that of general schedules, but we have been unable to prove it. However, we propose an exponential evaluation scheme whose complexity can be lowered as much as necessary, if only an estimation of the reliability is required. Simulation results allow us to assess the effectiveness of this method.

\subsection{Evaluation scheme}

The equation defining the reliability of a strict schedule (see Section 3, Equation (3)) cannot directly be expanded for evaluating the reliability of a strict schedule with fail-stop failures, because events $R_{i j}$ are no longer independent. This is why we propose an alternative formulation of $\operatorname{rel}(\pi)$ using the event $G_{i}$ defined as follows.

Let $G_{i}$ be the event that all tasks with an index lower than $i$ have at least one correct replica. Then, $G_{0}$ always occurs and $G_{i}$ is defined recursively for $i \geq 1$ as $G_{i}=\overline{\bigcap_{j} \overline{R_{i j}}} \cap$ $G_{i-1}$. Event $G_{i}$ occurs if and only if at least one processor $p_{j} \in P$ does not fail during the execution of its replica $t_{i}^{j}$, and if each of the first $i-1$ tasks has been successfully processed. As tasks are numbered according to some topological order, all the precedence constraints of $t_{i}$ are satisfied if $G_{i-1}$ occurs.
This latter formulation allows us to obtain a recursive expression for evaluating $\operatorname{rel}(\pi)$. Because the complexity of the evaluation scheme is exponential in the number $m$ of processors, we propose to control this complexity by limiting the scope of the recursive evaluations. The price to pay is that we have only an estimation of the reliability instead of the exact value.

We now state that the reliability of the schedule is given by $\operatorname{rel}(\pi)=\operatorname{Pr}\left[G_{n}\right]$. In order to obtain useful derivations, we introduce an event $\mathscr{E}$, which is an arbitrary intersection of events $\overline{R_{i j}}$. Let $\mathscr{E}^{\prime}=\bigcap_{j} \overline{R_{i j}} \cap \mathscr{E}$. Then, the calculation of $\operatorname{Pr}\left[G_{i} \mid \mathscr{E}\right]$ depends on $\operatorname{Pr}\left[G_{i-1} \mid \mathscr{E}\right], \operatorname{Pr}\left[G_{i-1} \mid \mathscr{E}^{\prime}\right]$ and on some elementary probabilities, i.e., $\operatorname{Pr}\left[\overline{R_{i j}} \mid \mathscr{E}\right]$ :

$$
\begin{aligned}
& \operatorname{Pr}\left[G_{i} \mid \mathscr{E}\right]=\operatorname{Pr}\left[\overline{\bigcap_{j} \overline{R_{i j}}} \cap G_{i-1} \mid \mathscr{E}\right] \\
& =\operatorname{Pr}\left[\overline{\bigcap_{j} \overline{R_{i j}}} \mid G_{i-1} \cap \mathscr{E}\right] \times \operatorname{Pr}\left[G_{i-1} \mid \mathscr{E}\right] \\
& =\left(1-\operatorname{Pr}\left[\bigcap_{j} \overline{R_{i j}} \mid G_{i-1} \cap \mathscr{E}\right]\right) \times \operatorname{Pr}\left[G_{i-1} \mid \mathscr{E}\right] \\
& =\left(1-\frac{\operatorname{Pr}\left[\bigcap_{j} \overline{R_{i j}} \cap G_{i-1} \mid \mathscr{E}\right]}{\operatorname{Pr}\left[G_{i-1} \mid \mathscr{E}\right]}\right) \times \operatorname{Pr}\left[G_{i-1} \mid \mathscr{E}\right] \\
& =\left(1-\frac{\operatorname{Pr}\left[G_{i-1} \mid \bigcap_{j} \overline{R_{i j}} \cap \mathscr{E}\right]}{\operatorname{Pr}\left[G_{i-1} \mid \mathscr{E}\right]} \operatorname{Pr}\left[\bigcap_{j} \overline{R_{i j}} \mid \mathscr{E}\right]\right) \times \operatorname{Pr}\left[G_{i-1} \mid \mathscr{E}\right] \\
& =\left(1-\frac{\operatorname{Pr}\left[G_{i-1} \mid \mathscr{E}^{\prime}\right]}{\operatorname{Pr}\left[G_{i-1} \mid \mathscr{E}\right]} \operatorname{Pr}\left[\bigcap_{j} \overline{R_{i j}} \mid \mathscr{E}\right]\right) \times \operatorname{Pr}\left[G_{i-1} \mid \mathscr{E}\right] \\
& =\left(1-\frac{\operatorname{Pr}\left[G_{i-1} \mid \mathscr{E}^{\prime}\right]}{\operatorname{Pr}\left[G_{i-1} \mid \mathscr{E}\right]} \prod_{j} \operatorname{Pr}\left[\overline{R_{i j}} \mid \mathscr{E}\right]\right) \times \operatorname{Pr}\left[G_{i-1} \mid \mathscr{E}\right]
\end{aligned}
$$

The last line is obtained by observing that all the events of the intersection $\bigcap_{j} \overline{R_{i j}}$ concern distinct processors and are independent.

Note that $\operatorname{Pr}\left[G_{0} \mid \mathscr{E}\right]=1$ for all $\mathscr{E}$ because $G_{0}$ always occurs. Therefore, $\operatorname{Pr}\left[G_{n}\right]$ can be computed recursively.

Before analyzing the complexity of this evaluation scheme, we introduce a mechanism for simplifying intersections of events $\overline{R_{i j}}$. Indeed, any event $\mathscr{E}=\overline{R_{i j}} \cap \overline{R_{i^{\prime} j}} \cap \ldots$ which is the intersection of at least two events $\overline{R_{\cdot j}}$ concerning the same processor $p_{j}$ can be reduced to a more concise definition. Only one event per processor is needed: with fail-stop failures, as soon as a processor has failed, it remains down until the end of the schedule. Hence, the event $\overline{R_{\cdot j}}$ which concerns the first task scheduled on $p_{j}$ is the only one to be considered for each processor $p_{j} \in P$. Consequently, we 
never compute any probability $\operatorname{Pr}\left[G_{i} \mid \mathscr{E}\right]$ where $\mathscr{E}$ is an intersection of more than $m$ events.

The complexity of recursive evaluation is $O\left(n^{m+1}\right)$. Indeed, there are $n$ events $G_{i}$ and for each of them, there are $(n+1)^{m}$ distinct intersections $\mathscr{E}$ (at most $m$ elements, and each may concern any of the $n$ tasks). We propose to control the exponent of the complexity cost by making some estimations. We limit the size of any intersection $\mathscr{E}$ to $k$ events. This is done by removing some of the events $\overline{R_{i j}}$ from $\mathscr{E}$ when the size of the intersection grows too large. Formally, we estimate that any new intersection $\mathscr{E}^{\prime}$ is equal to the intersection of at most $k$ events among $\bigcap_{j} \overline{R_{i j}} \cap \mathscr{E}$. Several choices are possible. We either select the remaining $k$ events arbitrarily, or we apply some heuristic procedure. As an example, we may be interested by selecting the subset of size $k$ that gives the lowest probability for $\operatorname{Pr}\left[G_{i} \mid \mathscr{E}^{\prime}\right]$. This heuristic is supported by the bound $\operatorname{Pr}\left[G_{i} \mid \mathscr{E} \cap \overline{R_{i j}}\right] \leq \operatorname{Pr}\left[G_{i} \mid \mathscr{E}\right]$ and would locally minimize the error done in the estimation. It provides a lower bound of the reliability for $k=0$ and an exact value for $k=m$ (an upper bound can be obtained by considering fail-stop failures as transient ones). For other values of $k$, however, we have no guarantee. The resulting complexity drops down to $O\left(n^{k+1}\right)$ with $k \in[0 . . m]$.

This reformulation of the reliability, and the derivations that follow, still end up with an exponential time estimation scheme. Another approach, still exponential, consists in considering all the possible choices (see the proof of Lemma 1 for further details on this approach). To the best of our knowledge, we are not aware of any procedure for evaluating the reliability of strict schedules with fail-stop failures in polynomial time (even when restricting the workload to independent tasks or to chain of tasks). We conjecture that this problem is \#P'-Complete just as it is the case for general schedules.

\subsection{Simulation results}

Simulations were conducted in order to assess this evaluation method. For each simulation, a random DAG is generated with 20 tasks, 30 edges (plus some edges to ensure that both the source and the sink are unique). Each cost is unitary and the platform consists of five homogeneous processors with Poissonian failure rates uniformly drawn from the interval $[0,0.05]$. Each task is scheduled twice on randomly chosen processors. On total, 300 schedules are obtained and their exact reliabilities lie in the range $[0.2,1]$. Note that due to the high cost of the approach, increasing the number of processors or the number of tasks makes it intractable to test each possible value for the parameter $k$. The chosen values enable each simulation to last less than one hour.

Fig. 6 depicts the running times of the method (black points) for each chosen value $k$. As expected, times grow

\section{Recursive evaluation scheme performance}

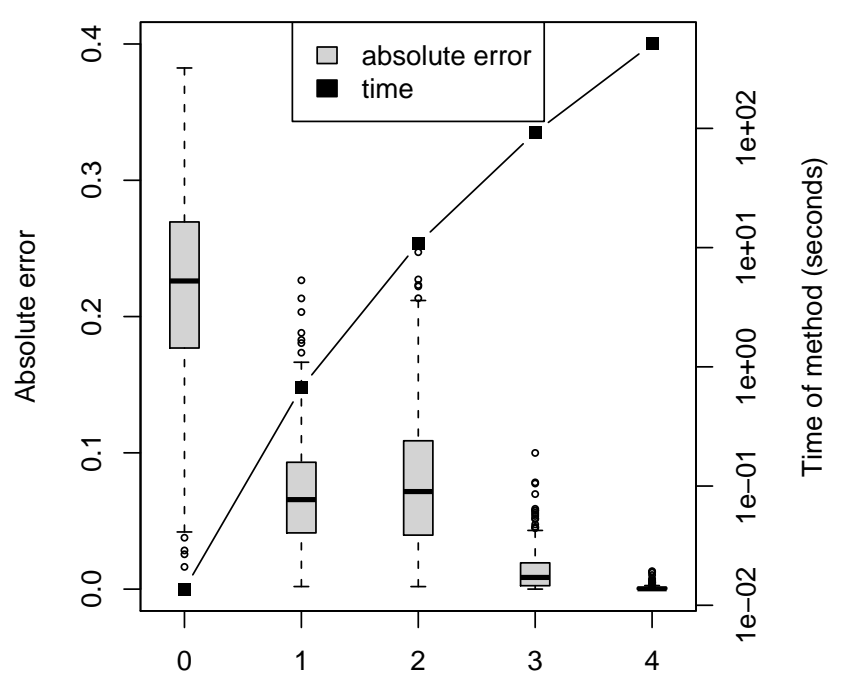

Number of considered events $\mathrm{k}$ in the evaluation

Fig. 6: Simulation results (20 tasks on five processors).

exponentially with $k$. For each value of $k$, the absolute difference between the true reliability and the estimated one is represented with boxplots. In a boxplot, the bold line is the median, the box shows the quartiles, the bars show the whiskers ( 1.5 times the interquartile range from the box) and additional points are outliers. We see that increasing $k$ leads to more precise results except for $k=2$. For $k=3$, the median is $0.86 \%$.

The method proposed in this section provides an estimation of the reliability of a strict schedule when failures are fail-stop. The precision of this estimation increases with the value of $k$. For high values of $k$, the method produces accurate results.

\section{Conclusion}

Fig. 2 summarizes known results on the complexity of reliability evaluation. The \#P'-Completeness of evaluating the reliability of general schedules holds true both for transient and for fail-stop failures, and constitutes the major contribution of the paper. Moreover, this result holds for more general cases such as for preemptive schedules and schedules with communications. While the strict/fail-stop combination remains open, we have provided a method to estimate the reliability while limiting evaluation costs, from which bounds can be derived (with $k=0$ ).

Future work will be devoted to close the complexity gap. We conjecture that the strict/fail-stop combination is \#P'Complete too, but we have been unable to prove it. An important research direction is to provide guaranteed approx- 
imations for the general case, with either failure type: can we derive a procedure to approximate the reliability within a prescribed bound, while limiting the evaluation time to some polynomial function of the application/platform parameters? Finally, we plan to study methods for effectively constructing reliable schedules based on a relevant evaluation mechanism. Whereas the first step would be to develop static scheduling algorithms, dynamic strategies could also provide interesting insights.

Acknowledgment: This work was supported in part by the ANR StochaGrid and RESCUE projects, and by the INRIA $A L E A E$ project. We would like to thank the associate editor and the reviewers for their comments and suggestions, which greatly improved the final version of this paper.

\section{References}

Awerbuch B., Azar Y., Fiat A., Leighton F. T. (1996) Making commitments in the face of uncertainty: How to pick a winner almost every time. In: 28th ACM SToC, pp 519-530

Bannister J., Trivedi K. S. (1983) Task allocation in fault-tolerant distributed systems. Acta Informatica 20:261-281

Barlow R. E., Proschan F. (1967) Mathematical theory of reliability. John Wiley, New York

Bhatt S., Chung F., Leighton F., Rosenberg A. (1997) On optimal strategies for cycle-stealing in networks of workstations. IEEE Trans Computers 46(5):545-557

Bodlaender H. L., Wolle T. (2004) A note on the complexity of network reliability problems. IEEE Trans Inf Theory 47:1971-1988

Bream B. (1995) Reliability Block Diagrams and Reliability Modeling. Tech. rep., Office of Safety and Mission Assurance, NASA Lewis Research Center

Brucker P. (2004) Scheduling Algorithms. Springer-Verlag

Cappello F., Geist A., Gropp B., Kale L., Kramer B., Snir M. (2009) Toward Exascale Resilience. Int Journal of High Performance Computing Applications 23(4):374-388

Dongarra J., Jeannot E., Saule E., Shi Z. (2007) Bi-objective Scheduling Algorithms for Optimizing Makespan and Reliability on Heterogeneous Systems. In: 19th ACM Symp. on Parallelism in Algo. and Archi. (SPAA'07), San Diego, CA, USA

Girault A., Kalla H. (2009) A novel bicriteria scheduling heuristic providing a guaranteed global system failure rate. IEEE Trans Dependable Secure Computing 6(4):241-254

Girault A., Saule E., Trystram D. (2009) Reliability versus performance for critical applications. J Parallel and Distributed Computing 69(3):326-336

Jeannot E., Saule E., Trystram D. (2008) Bi-Objective Approximation Scheme for Makespan and Reliability Optimization on Uniform Parallel Machines. In: The 14th Int. Euro-Par Conf. on Parallel and Distributed Computing, Spain

Kartik S., Murthy C. S. R. (1997) Task allocation algorithms for maximizing reliability of distributed computing systems. IEEE Trans Computers 46(6):719-724

Provan J. S., Ball M. O. (1983) The complexity of counting cuts and of computing the probability that a graph is connected. SIAM J Comp 12(4):777-788

Rosenberg A. L. (2002) Optimal schedules for cycle-stealing in a network of workstations with a bag-of-tasks workload. IEEE Trans Parallel Distrib Syst 13(2):179-191
Shatz S., Wang J. (1989) Models and algorithms for reliability-oriented task-allocation in redundant distributed-computer systems. IEEE Trans Reliability 38(1):16-26

Shatz S., Wang J., Goto M. (1992) Task allocation for maximizing reliability of distributed computer systems. IEEE Trans Computers 41(9):1156-1168

Valiant L. G. (1979) The complexity of enumeration and reliability problems. SIAM J Comput 8(3):410-421

Zhu D., Melhem R., Mossé D. (2004) The effects of energy management on reliability in real-time embedded systems. In: International Conference on Computer Aided Design, ICCAD'04, San Jose (CA), USA, pp 35-40 